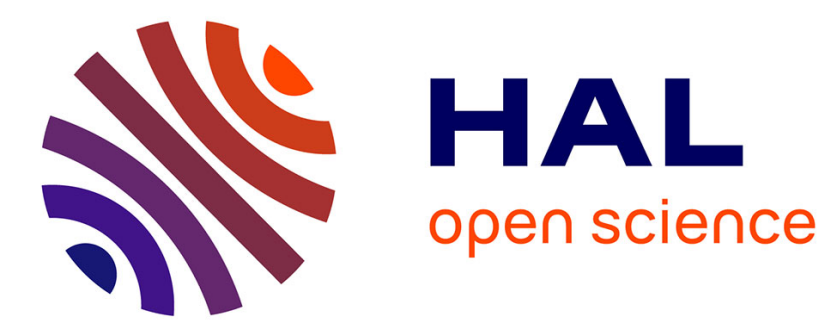

\title{
IMMERSION-TYPE TRANSMISSION ELLIPSOMETRY OF VERY THIN METAL FILMS
}

\author{
M. Yamamoto, K. Ichiji, Y. Wada, K. Takeuchi, K. Kinosita
}

\section{To cite this version:}

M. Yamamoto, K. Ichiji, Y. Wada, K. Takeuchi, K. Kinosita. IMMERSION-TYPE TRANSMISSION ELLIPSOMETRY OF VERY THIN METAL FILMS. Journal de Physique Colloques, 1983, 44 (C10), pp.C10-383-C10-386. 10.1051/jphyscol:19831078 . jpa-00223536

\section{HAL Id: jpa-00223536 https://hal.science/jpa-00223536}

Submitted on 1 Jan 1983

HAL is a multi-disciplinary open access archive for the deposit and dissemination of scientific research documents, whether they are published or not. The documents may come from teaching and research institutions in France or abroad, or from public or private research centers.
L'archive ouverte pluridisciplinaire HAL, est destinée au dépôt et à la diffusion de documents scientifiques de niveau recherche, publiés ou non, émanant des établissements d'enseignement et de recherche français ou étrangers, des laboratoires publics ou privés. 


\title{
IMMERSION-TYPE TRANSMISSION ELLIPSOMETRY OF VERY THIN METAL FILMS
}

\author{
M. Yamamoto*, K. Ichiji, Y. Wada, K. Takeuchi and K. Kinosita \\ Department of Physics, Gakushuin University, Mejiro, Toshima-ku, Tokyo 171, \\ Jiopan \\ * Research Institute for Scientific Measurements, Tohoku University, \\ Sendai 980 , Japan
}

\begin{abstract}
Résumé - Une couche mince de métal est déposée sur la face la plus longue d'un prisme rectangulaire d'indice $n_{0}$. Cette couche est recouverte par un film mince transparent de liquide du même indice $n_{0}$, puis est prise en "sandwich" en plaçant par-dessus un autre prisme identique au premier. On détermine ainsi le rapport des amplitudes complexes transmises, en polarisation $p$ et $s$, par le film qui est ainsi "suspendu" dans un milieu uniforme d'indice $n_{0}$, ce qui permet d'évaluer les constantes optiques de ce film.
\end{abstract}

\begin{abstract}
The metal film is deposited on the longer face of a rectangular prism of index $n_{0}$. The film is covered by a thin, transparent liquid film of index $n_{0}$, and is sandwiched by placing another, identical prism on top of $i t$. The ratio in complex amplitude transmissivity for the p- and s-polarized light of the film thus optically "suspended" in a uniform medium of index $n_{0}$ is determined, from which the optical constants of the film are evaluated.
\end{abstract}

\section{§1. Introduction}

The idea of the immersion-type transmission ellipsometry (ITE) described here was born when we were searching for an efficient method for the study of the optical properties of vacuum-deposited metal films in their earliest growth stages. The orthodox spectral acisorption measurement is hardly feasible with such films that are almost tranisparent: these "films" consists of separate atom clusters a few tenths to a few nanometers in diameter scattered over the substrate surface. We had found that reflection ellipsomeiry (RE) was a useful means for the study of such films $/ 1 /$, but the uncertainties in interpreting its data drove us to the search for an independent method that compared with it. ITE was our first choice.

In ITE, the $\mathrm{fi} / \mathrm{m}$ is deposited on a transparent substrate of index $n_{0}$, the space above the films is filled with a material of the same index, and the ratio in amplitude transmissivity of the $p$ - and s-polarized light at an angle of incidence $\phi$, $\tilde{t}_{p} / \tilde{t}_{s}$, is determined for the film thus optically "suspended" in a uniform medium of index $n_{0}$. Let us write

$$
\tilde{t}_{p} / \tilde{t}_{s}=\tan \Psi_{t} \cdot \exp \left(i \Delta_{t}\right)
$$

after the usual expression $\tilde{r}_{p} / \tilde{r}_{S}=\tan \psi \cdot \exp (i \Delta)$. We can easily derive that, under conditions given above,

Here

$$
\tan \Psi_{t} \cdot \exp \left(i \Delta_{t}\right)=\frac{1-\tilde{r}_{p}^{2}}{1-\tilde{x}_{s}^{2}} \cdot \frac{1-\tilde{r}_{s}^{2} \exp (i 2 \beta)}{1-\tilde{r}_{p}^{2} \exp (i 2 \beta)}
$$

$$
\beta=-2 \pi(d / \lambda)\left(\tilde{n}^{2}-n_{0}^{2} \sin ^{2} \phi\right)^{\frac{1}{2}},
$$


$\tilde{r}_{p}$ and $\tilde{r}_{s}$ are the Fresnel coefficients at the two film surface, and $d$ and $\tilde{n}=n$ - $i k$ are the thickness and complex refractive index of the film respectively. Since eq. (2) is an equation of the first degree with respect of $\exp (i 2 \beta)$, the evaluation of $n$ is much simpler in ITE than in conventional RE, as will be seen in $\$ 3$.

To realize the situation described above experimentally, it is convenient to deposit the film on a rectangular prism of index $n_{0}$, lay a small drop of transparent liquid of index $n_{0}$ on the fi $7 \mathrm{~m}$, and place another, identical prism on top of it (see $S$ in Fig. 1). The space between the metal film and the second prism is thus filled with a very thin liquid $\mathrm{film}$ of index $n_{0}$.

Some details of our experimental procedure are given in $\$ 2$. Preliminary results with Au films are given in $\$ 3$, and are discussed in $\$ 4$ together with some open questions concerning the permeation of the matched-index liquid into the granular metal film.

\section{§2. Experimental Procedure}

Figure 1 shows schematically the extinction type ellipsometer for use in ITE. Up to now, most measurements have been carried out with He-Ne laser light.

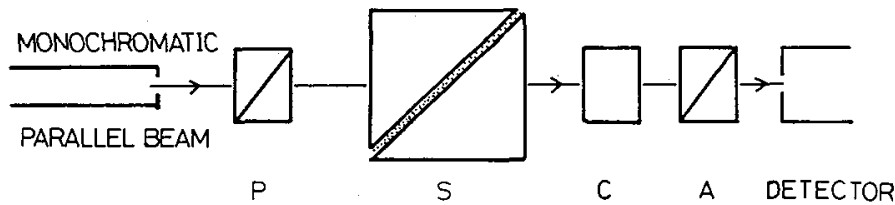

Fig. 1 - Optical system for ITE. P, A: Glan-Thompson prisms. $C: \lambda / 4-p l a t e$ S: sample complex (see $\S 1$ ).

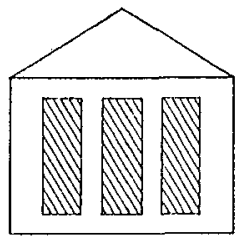

Fig. 2 - Metal films deposited on prism face.
Three pieces of $\mathrm{fi} 7 \mathrm{~m}$, usually of different thicknesses,were vacuum-deposited on the longer face of a glass rectangular prism of index $n_{0}=1.515$ (Fig. 2). In some cases, $\Delta$ and $\psi$ were determined for each film by a reflection ellipsometer $/ 1 /$ before breaking the vacuum (stage A). After breaking the vacuum, the metal films were sandwiched between the prisms, together with the matched-index liquid, as was described in §1. The Tiquid was prepared by mixing silicone oils HIVAC F-4 and KF-54 (Shin-Etsu Chemical Co., Ltd.) so that its index was equal to $1.515 \pm 0.001$ at $20^{\circ} \mathrm{C}$ (the temperature coefficient is $\left.-5 \times 10^{-4} /{ }^{\circ} \mathrm{C}\right)$. The measurement of $\Delta_{t}$ and $\Psi_{t}$ was first

carried out immediately after sandwiching the film (within 30 minutes) when the liquid did not seem to have started permeation into the metal films (Stage B). The measurement was repeated after the granular metal films had been completely permeated by the liquid (Stage $\mathrm{C}$ ). A11 these ITE measurements were made keeping the sample complex S (Fig. 1) at $20^{\circ} \mathrm{C}$.

The permeation started at the periphery of the fi $1 \mathrm{~m}$ and proceeded toward the centre. The permeated region could easily be distinguished by a marked reduction in reflectivity. The boundary line between the permeated and unpermeated regions was quite distinct. It took a few weeks for the $f i 7 m$ to be completely permeated.

\section{§3. Preliminary Results with Au Films}

Figure 3 shows the changes in $\Delta_{t}$ and $\Psi_{t}$ with the film thickness $d$. Curves $B_{1}$ and $C_{1}$ represent the observations at stages $B$ and $C$ respectively

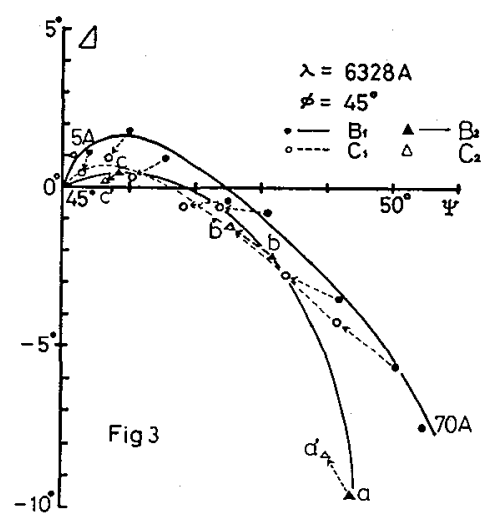

Fig. 3 -See text for detail. 
for $\mathrm{Au}$ films evaporated in an oil-diffusion-pumped system $\left(10^{-3} \mathrm{~Pa}\right)$. Curve $\mathrm{B}_{2}$ has tentatively been drawn through points $a, b$ and $c$ that represent the observations at stage $B$ for three $A u$ films evaporated in an ion-pumped system $(10-5 \mathrm{~Pa})$. The $\left(\Delta_{t}\right.$, $\left.\Psi_{t}\right)$ values for these films at stage $C$ are given by points $a^{\prime}, b^{\prime}$ and $c^{\prime}$.

The parameters $\tilde{n}=n-i k$ and $d$ of the effective fizm, or the hypothetical homogeneous $f j l m$ optically equivalent to the two-dimensional array of Au particles, can be evaluated as follows. First we substitute the observed values of $\Delta t$ and $\Psi_{t}$ into eq. (2), and solve it for $\exp (i 2 \beta)$. If we raise the obtained expression for $\exp (i 2 \beta)$ to the $(2 \pi d / \lambda \beta)$-th power, we obtain an equation of the form

$$
\exp (i 4 \pi d / \lambda)=f\left(\tilde{n}, n_{0}, \Delta_{t}, \psi_{t}\right),
$$

from which it follows that

$$
\left|f\left(\tilde{n}, n_{0}, \Delta_{t}, \psi_{t}\right)\right|^{2}=1 \text {. }
$$

Equation (4) determines a curve on the $n-k$ plane for each pair of observed values of $\Delta_{t}$ and $\Psi_{t}$. Each point on the curve corresponds to a value of $d$ that can be calcu-

lated according to eq. (4).

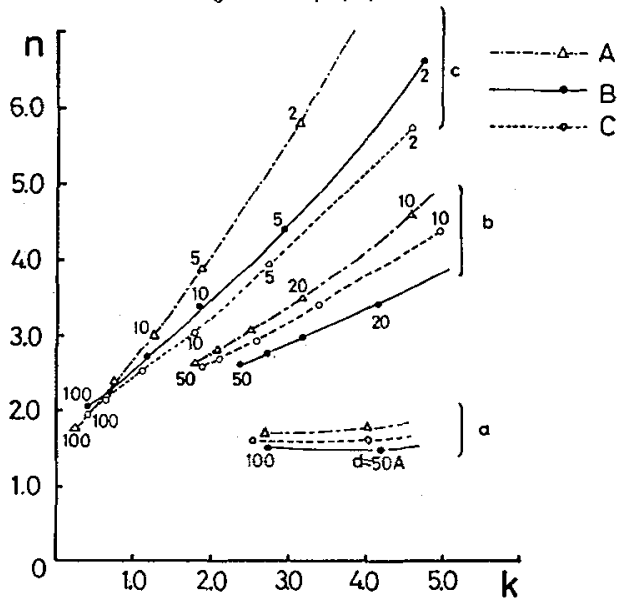

Fig.4- Projected loci of the possible $(n, k, d)$ combinations for some data points in Fig. 3.
In Fig. 4, the solid curves represent the $n-k$ curves for points $a, b$ and $c$ (stage B), and the broken ones those for points $a^{\prime}, b^{\prime}$ and $c^{\prime}$ (stage $c$ ). The chain lines give the values of $n$ and $k$ for the three films at stage $A$ (before breaking the vacuum) that have been calculated from the values of $\Delta$ and $\psi$ obtained by in-situ reflection ellipsometry.

\section{§4. Discussion}

Before starting the experiment, we did not expect that the permeation of the matched-index liquid into granular metal films with thicknesses of the order of nanometers would take a few weeks. This, in itself, is an interesting problem in surface physics, but at the moment we will concentrate on the interpretation of the ellipsometric data.

Let us suppose that at stage B there was empty space between the substrate and the liquid surface, the latter being in direct contact only with the top surfaces of the metal particles, whereas at stage $C$ the entire space between the particles was completely filled with the liquid. If we apply the Bruggeman EMA theory $/ 2 /$ to these models, we have

$$
(1-q) \frac{1-\tilde{n}_{\mathrm{B}}^{2}}{1+2 \tilde{n}_{\mathrm{B}}^{2}}+q \frac{\tilde{N}^{2}-\tilde{n}_{\mathrm{B}}^{2}}{\widetilde{N}^{2}+2 \tilde{n}_{\mathrm{B}}^{2}}=0
$$

for stage $B$, and

$$
(1-q) \frac{1.515^{2}-\tilde{n}^{2} C}{1.515^{2}+2 \tilde{n}_{C}^{2}}+q \frac{\tilde{N}^{2}-\tilde{n}_{C}^{2}}{\tilde{N}^{2}+2 \tilde{n}_{C}^{2}}=0
$$

for stage $C$, where $\tilde{n}_{B}$ and $\tilde{n}_{C}$ are the refractive indices of the effective film at respective stages, $\tilde{N}$ is the refractive index of the metal particles, and $q$ is the filling factor. Substituting a wide range of values of $\tilde{n}_{B}$ and $\tilde{n}_{C}$ (Fig. 4) that correspond to point $b$ in Fig. 3 into eqs. (5) and (6), we searched for the solutions $q$ and $\tilde{N}$ that satisfy the conditions

$$
I_{m}(q)=0,0<q<1 ; \operatorname{Re}(\tilde{N})>0, I_{m}(\tilde{N}) \leq 0
$$


No such solutions were obtainable as far as we assumed that the effective film thickness $d$ at stage $C, d_{C}$, was identical with that at stage $B, d_{B}$.

It would be more natural, however, to assume that $d$ changes with the liquid permeation. Accordingly, we chose a thickness $d_{B}$ on the $n-k$ curve for $b$ in Fig. 4 which was compatible with the mass thickness $d_{m}$ of this film $(1.2 \mathrm{~nm})$, and searched for the value of $d_{C}$ on the curve for $b^{\prime}$ which, combined with the assumed value of $a_{B}$, can give $q$ and $\tilde{N}$ satisfying the conditions (7). Repeating such calculations, we were able to find a range of plausible solutions. A typical set of such solutions are:

$$
d_{\mathrm{B}}=4.0 \mathrm{~nm}, d_{\mathrm{C}}=2.0 \mathrm{~nm}, \quad q=0.33, \tilde{N}=0.6-17 i
$$

The range of $d_{B}$ that can give meaningful solutions for this particular film is $3-5$ $\mathrm{nm}$. Any value of $d_{B}$ in this range gave q00.33: The value $q$ estimated from the electron micrograph of this film (Fig. 5) on the assumption that the particles were hemicylindrical was 0.36 .

Thus we conclude that (1) just after the metal film has been sandwitched with the matched-index 1 iquid there remains empty space between the substrate and the liquid surface, and (2) when completely permeated by the liquid the effective film thickness is reduced to about one half of its original value.

\section{References}

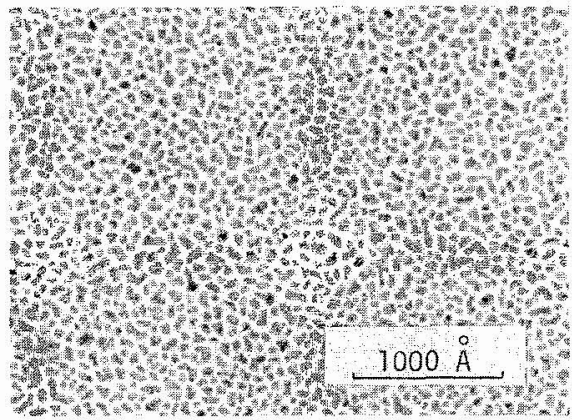

Fig. 5 Electronmicrograph of the sample b shown in Fig. 3.

1. K. Kinosita, M. Yamamoto, T. Gotoh, S. Hirasawa, Y. Tomohiro and S. Kawabata, Proc. 4th Int. Conf. Solid Surfaces, Cannes, 1980, pp. 696-698.

K. Kinosita, M. Yamamoto and S. Hirasawa, Thin Solid Films 90 (1982) 19.

2. D. A. G. Bruggemann, Ann. Phys. (Leipzig), 24 (1935) 636 